\title{
Chemomechanical Removal of Caries - An Invasive Method as Extension for Prevention: A Review
}

\author{
Arka Prova Bhattacharjee ${ }^{1}$, Deepthi Nirmal Gavarraju ${ }^{2}$, Yesh Sharma ${ }^{3}$, Shalini Singh ${ }^{4}$, Kritika Sehrawat ${ }^{5}$, Rahul \\ VC. Tiwari ${ }^{6}$ \\ ${ }^{1}$ Department of Conservative Dentistry and Endodontic, Sibar Institute of Dental Sciences, Ntruhs, Guntur, A.P, India \\ ${ }^{2}$ Department of Pedodontics and Preventive Dentistry, Sibar Institute of Dental Sciences, Ntruhs, Guntur, A.P, India \\ ${ }^{3}$ Department of Conservative Dentistry and Endodontic, Maharaja Ganga Singh Dental College, Sri Ganga Nagar, Rajasthan, \\ India \\ ${ }^{4}$ Department of Conservative Dentistry and Endodontic, Vyas Dental College and Hospital, Jodhpur, Rajasthan, India \\ ${ }^{5}$ Department of Oral and Maxillofacial Surgery, Sudha Rustagi Dental College and Research Centre, Faridabad, Haryana, India \\ ${ }^{6}$ Department of Oral \& Maxillofacial Surgery and Dentistry, Jubilee Mission Medical College Hospital and Research Institute, \\ Thrissur, Kerala, India
}

Corresponding Author: Rahul VC. Tiwari, MD, Fellowship in Orthognathic Surgery, Department of Oral \& Maxillofacial Surgery and Dentistry, Jubilee Mission Medical College Hospital and Research Institute, Thrissur, Kerala-680005, India. Tel: +7034382238, E-mail: drrahulvctiwari@gmail.com

Received May 20, 2017; Accepted June 23, 2017; Online Published August 29, 2017

\begin{abstract}
The traditional treatment of caries was merely surgical. Conventionally, the only effective method of eliminating the disease was to completely remove all of the demineralized area of the tooth structure. Even the smallest area of demineralization required the excavation of a standard amount of sound tooth structure to prevent the progression of disease. Chemomechanical caries removal (CMCR) is a non-invasive technique in which chemical agents are used to eliminate the infected dentin. This dissolution-based caries removal method uses a chemical agent assisted by atraumatic mechanical vigor for the excavation of soft carious dentine. This technique was developed by Dr. G. V. Black as "extension for prevention" which led to specific of sound tooth structure. In later years, the profession moved toward practicing preventive dentistry and following more conservative and tooth-preserving procedures. This paper discusses newer invasive techniques for caries excavation.

Keywords: Caries; Chemomechanical; Removal

Citation: Bhattacharjee AP, Gavarraju DN, Sharma Y, Singh S, Sehrawat K, Tiwari RVC. Chemomechanical removal of caries An invasive method as extension for prevention: a review. Int J Med Rev. 2017;4(3):66-69. doi: 10.29252/ijmr-040302.
\end{abstract}

\section{Introduction}

The procedures used to remove caries can be classified clinically according to B. D. J. 2000 as: Mechanicalrotary, Mechanical-non-rotary, chemo-mechanical, photo-ablation, hand pieces + burs, hand excavation, air abrasion, ultrasonics, sonoabrasion, Caridex, Carisolv and enzymes, and lasers. Each of the above-mentioned techniques has its own claim to selectively removing demineralized dentin. An ideal method should fulfill certain factors to gratify both the clinician and the patient. It must have the capability of disseminating and excavating carious tooth structure only, be painless, and require only a minimum amount of pressure. Furthermore, it should not produce vibration or heating during the operation period, it should be reasonably priced, and it should be simple to maintain. ${ }^{1-3}$ The handpieces and burs are used universally, even with their obvious disadvantages, like sensitivity to vital pulp, applying pressure/heat to the tooth, and requiring local anesthetics. Eventually, the chemo-mechanical approach, an alternative non-invasive technique for removing caries, came into play.

With this technique, a chemical solution is applied onto the decayed tooth structure and allowed to soften the tissue, which is finally scraped with a blunt hand instrument. Many solutions have been introduced and marketed since the 1970's, and they will be discussed in detail in this article. Before discussing individual products, the layers present in carious dentin which are important in this paper with respect to the chemicals will be introduced. ${ }^{4,5}$

Copyright (C) 2017 The Author(s). This is an open-access article distributed under the terms of the Creative Commons Attribution License (http://creativecommons.org/licenses/by/4.0), which permits unrestricted use, distribution, and reproduction in any medium, provided the original work is properly cited. 
Carious Dentin and Its Chemomechanical Removal Carious dentin is comprised of an outer and an inner layer. The outer layer: Decalcified-degenerated collagen fibers, Infected-non-remineralizable, necrotic (This layer should be removed). The inner layer is between the outer and normal dentin, less decalcified, bacteria free, remineralized collagen fibers are present, and vital odontoblastic process is present. ${ }^{6}$ This layer should be left intact. Preferably, while preparing the decayed tooth, the clinician must only remove the outer carious layer of dentin while retaining the inner non-carious layer intact. ${ }^{7}$ The chemomechanical method claims to do so. Let us now see the different products available to us. Chemo-mechanical approach: Originally in 1972, the chemo-mechanical approach was introduced in the form of G.K. 101 solution. Goldman and Kronman (1976), in a study on the possibility of removing caries, reported the chemical use of GK-101 (NMG), which consists of Nmonochloro glycine (NMG) and sodium hypochlorite. Glycine was added to counteract the corrosive effect of $\mathrm{NaOCl}$, also called GK-101G. The mode of action of this chemical has been described as the chlorination of peptide bonds holding amino groups of protein-forming NMG compounds. NMG has the capacity to change hydroxyproline into pyrole-2-(glycine peptide carboxy glycine) which is an important factor in the chemomechanical removal of caries. Therefore, the halfdegraded collagen in the destroyed dentin was chlorinated using NMG solution, and this, in addition, affected the collagen (secondary and quaternary structure) by disturbing the hydrogen bond. In this manner the removal of destroyed tooth structure was facilitated. GK-101 (NMG) testing was done in bovine Achilles tendon collagen to observe what actually happens to the collagen fibers. SEM evaluation showed fraying fibrils, i.e. the vital structure was intact, but there was some disjunction in some peripheral fibers; spinaling fibrils, i.e. the attraction between nearby fibers was lost, and there was shortening of the individual fibers; dissociating fibrils, i.e. structure was thoroughly separated or isolated, and the fiber orientation was poor and difficult to characterize; and amorphous material, i.e. there was little definitive structure and material which was hard to define as collagen. The advantages were that it is painless and has no harmful effects on pulp. Kurosaki et al. and Brannstorm et al. in their respective studies showed that it removed only the outer diseased carious layer. They assumed that the demineralization may be due to a particular attack of the solution exclusively on degraded collagen fibers without distressing the sound inner layer and normal dentin beneath. The disadvantage was that the process was very slow. Later they found that the framework was more compelling if amino-butyric acid was used in place of glycine, which evolved in the GK-101E. This was approved by the FDA (Food and Drug Administration) in 1984 and commercialized as "CARIDEX" GK-101E, containing instead of NMG, "N-monochloro-DL-2- aminobutyric acid" (NMAB). The system consists of a reservoir, heater, pump, and a handpiece with an application tip in various shapes and sizes. In vitro studies done by Goldman et al. stated that Caridex removed both layers of caries, leaving behind sound dentin. Schertz et al. reported that a histological evaluation after Caridex was used exhibited $90 \%$ of caries with residual decay; therefore, they concluded that Caridex should be used with a spoon excavator..$^{8-10}$

\section{Theories}

A number of theories have been postulated as to why there is reduced pain ${ }^{1}$. They are:

a. Lack of cutting into caries-free dentin.

b. Relatively few dentinal tubes are exposed.

c. No vibrations from drilling.

d. No temperature variations.

e. Dentin is always covered with an isotonic gel at body temperature.

f. Psychologically quiet and less traumatic experience.

Indications are where conservation of tooth structure is essential, for example in removal of root/cervical caries, coronal caries management without cavitation, caries excavation from the crown and bridge abutment margins, completion of tunnel preparation, in cases where the use of local anesthesia is contraindicated, in anxious patients, in deciduous dentition, and as a traumatic restorative technique (ART). Its advantages over Caridex include: three amino groups are incorporated instead of one, because interaction and degradation efficiency is increased; Carisolv has higher viscosity, which allows for the application of a higher concentration of amino acids and $\mathrm{NaOCl}$ without increasing total volume or amount (only 0.2-1.0 ml Carisolv required as compared to $250-500 \mathrm{ml}$ of Caridex),its increased viscosity helps in precision placement, the gel does not need to be heated or supplied through a pump, and its improved shelf life.

\section{Review of Published Literature}

Clinical studies done by Zinek et al. showed 90-100\% removal of decay with Caridex, but it took a very long time. Rompen and Chorpentier found that Caridex was not bactericidal in 17 samples cultured from the decay. Yip et al. combined NMAB +2 urea in deciduous teeth and found it to be better. ${ }^{11}$ Pioch and Stachle investigated the shear strength at the DEJ after treatment with Caridex for adhesive and bonding systems. Caridex was found to decrease the shear strength at the DEJ in bovine teeth. This was attributed to the denaturation of the collagen. This disadvantage related to tooth fracture needs further clinical studies and investigations. Kurusaki et al., Walkman et al., and Wedenberg and Burnstein individually investigated the biocompatibility of Caridex to pulp, and they found it to be biocompatible. Because of the alkalinity of Caridex, it was found that below the necrotic zone, there was production of a hard tissue matrix. (Zones: transient, dark, body of lesion, 
surface.) Yip et al. investigated the mineralization of the dentinal surface remaining after the use of a small sample of Caridex. They used "back scattered electron imaging" (BSI) and "electron probe micro-analysis" (EPMA) to calculate the surface level of $\mathrm{Ca}$ and $\mathrm{P}$ ions. The authors concluded that the amount of $\mathrm{Ca}$ and $\mathrm{P}$ was $2: 1$, which matched that of sound dentin, because it is better. Other studies have reported that often with the usage of Caridex, the dentinal surface produced A high degree of roughness, undercuts, dentin scales, dentin tubules were partially patent, and a smear-free surface. It was postulated that it was better for adhesive restorative material without the necessity of acid etching. Zinck et al. also evaluated patient acceptance and found a 93\% acceptance level. Although Caridex had many advantages, it was very expensive, time consuming, had a bulky delivery system, needed additional mechanical means to remove decay, and required large volumes of solutions (200-500 ml). Following this, a gel-based system was introduced in collaboration with Mediteam (Dental attracting: Gotiberg AB) in 1998 called Carisolv (Dental update: 2000). Carisolv was initially approved for clinical use in dental practice by the Swedish counterpart to the U.S. FDA. ${ }^{12}$ Its composition is as follows: the formulation is isotonic in nature and consists of 2 syringes. The syringes were $0.5 \% \mathrm{NaOCl}$, syringe 3 amino acids, glutamic, Lucine, Lysine; gel substance carboxy methyl cellulose, sodium chloride, sodium hydroxide, erythrosine to make the gel visible, and saline solution (i.e. colonizing indicator). Mode of action: Carisolv is alkaline in nature with a $\mathrm{pH}$ of around 11. Upon mixing, the positively and negatively charged groups of amino acids become chlorinated due to the presence of $\mathrm{NaOCl}$ and its constituents. This leads to interaction with dentin, which involves the proteolytic degradation rather than demineralization of collagen, softening and removing the carious altered dentin and preserving the sound dentin. The gel consistency permits the dynamic particles access to the dentin for a more extended period than the equivalent irrigating solution in the Caridex system. This gel also helps by lubricating the hand instrument specifically designed for Carisolv. The instrument consists of 4 different handles with 8 interchangeable tips ranging in size from $0.3 \mathrm{~mm}-2 \mathrm{~mm}$. These instruments resemble excavators, but they are designed to be used in rapid whisking or curetting fashion, thereby being limited to only diseased tissue. ${ }^{13-16}$ The configuration of the instrument allows access to all areas of the lesion and helps give a tactile sensation and in differentiating between carious and non-carious dentin. Furthermore, it helps apply the gel.

Cavity preparation: The two syringes ought to be blended just prior to use, as its efficiency decreases after 20-30 minutes. The two gels are mixed until a uniform color is obtained in a dappen dish. The mixed gel is then applied to the demineralized lesion and left in place for 30 seconds to allow it to degrade the diseased dentin before instrumentation. Rapid, light pressure is applied with the instrument to facilitate caries removal. As the caries is removed, the gel becomes cloudy with debris, indicating cleaning with water. ${ }^{17} \mathrm{Gel}$ is applied again for further removal. Assessment (i.e. when to stop): It is time to stop when the gel is no longer cloudy. Tactile sensation on the carious dentin will present with "a catch", whereas in a sound dentin the instrument will pass easily. After completion, the cavity appears frosted and irregular compared to the smooth preparation of the conventional bur and handpiece. The reason for this visual difference is that in a conventional preparation the presence of a smear layer, which is over the underlying dentin, gives a smooth, glossy appearance. In contrast, chemomechanically treated dentin lacks a smear layer and also forms an irregular dentin layer, giving it a matte finish. Patient's acceptance: This method takes a shorter amount of time and results in no pain or discomfort. In vitro studies: Jepsen et al. analyzed the collagen structure of residual dentin after the use of Carisolv. They found that it differed from sound dentin and had characteristic denatured collagen. ${ }^{18}$ In clinical studies: From the abstracts published in Stockholm, different authors concluded individually that although Carisolv removed $99 \%$ of decay, it was slower than conventional techniques. Enzymes: Goldberg and Keil (1989) conducted a study on the removal of caries using enzymes and successfully removed soft demineralized dentin without affecting the sound dentin using bacterial achromobacter collagenase. Enzyme pronase, which is a non-specific proteolytic enzyme originating from streptomyces griseus, also helps in removing caries. Experiments are still ongoing for the validity of such enzyme. ${ }^{19,20}$

\section{Conclusions}

As with all new procedures, there are the uncertainties of learning new techniques, using new techniques, clinical time, cost, etc., all of which need to be considered on an individual level. Over the decades, dentistry has changed dramatically, and practitioners follow new trends and master new techniques. The chemomechanical technique removes less sound tooth structure and reduces pulpal irritation. This technique can help supplement and complement other methods of caries removal, but further research and clinically-based experiments are required for the acceptance of such new techniques.

\section{Authors' Contributions}

APB: Abstract and Introduction; DNG: Carious dentin and its chemomechanical removal; YS: Carious dentin and its chemomechanical removal; SS: Review of published literature; KS: Review of published literature; RVC: Conclusion and References.

\section{Conflict of Interest Disclosures}

All authors declare that they have no conflicts of interest.

\section{Funding}

None. 


\section{References}

1. Morrow LA, Hassall DC, Watts DC, Wilson NH. A chemomechanical method for caries removal. Dent Update. 2000;27(8):398-401. doi: 10.12968/denu.2000.27.8.398. pmid: 11218535.

2. Motta LJ, Martins MD, Porta KP, Bussadori SK. Aesthetic restoration of deciduous anterior teeth after removal of carious tissue with Papacarie. Indian J Dent Res. 2009;20(1):117-20. pmid: 19336873.

3. Banerjee A, Watson TF, Kidd EA. Dentine caries excavation: a review of current clinical techniques. $\mathrm{Br}$ Dent J. 2000;188(9):476-82. pmid: 10859846.

4. Beeley JA, Yip HK, Stevenson AG. Chemochemical caries removal: a review of the techniques and latest developments. Br Dent J. 2000;188(8):427-30. pmid: 10953400

5. Frencken JE, Pilot $T$, Songpaisan $Y$, Phantumvanit $P$. Atraumatic restorative treatment (ART): rationale, technique, and development. J Public Health Dent. 1996;56(3 Spec No):135-40; discussion 61-3. pmid: 8915958.

6. Goldman $\mathrm{M}$, Kronman $\mathrm{JH}$. A preliminary report on a chemomechanical means of removing caries. J Am Dent Assoc. 1976;93(6):1149-53. pmid: 794090.

7. Zinck JH, McInnes-Ledoux P, Capdeboscq C, Weinberg R. Chemomechanical caries removal?a clinical evaluation. J Oral Rehabil. 1988;15(1):23-33. doi: 10.1111/j.1365-2842. 1988.tb00143.x.

8. Bussadori SK, Castro LC, Galvao AC. Papain gel: a new chemo-mechanical caries removal agent. J Clin Pediatr Dent. 2005;30(2):115-9. pmid: 16491964.

9. Hadley J, Young DA, Eversole LR, Gornbein JA. A laserpowered hydrokinetic system for caries removal and cavity preparation. J Am Dent Assoc. 2000;131(6):777-85. pmid: 10860330.

10. Carnerio F, Nadanovisky P. Remocaoquimicomecanica da carie. In: Carnerio F, Nadanovisky P, editors. Dentistica ultraconservative. Sao Paulo: Paulo:Santos 2003. p. 59-71.

11. Yip H, Beeley J. Studies on the reaction of $\mathrm{NaHCl}$ and NMAB with collagen. J Dent Res. 1989;68:982.

12. Moran C, Lynch E, Petersson L, Borsboom P. Comparison of caries removal using Carisolv or a conventional slow-speed rotary instrument. Caries Res. 1999;33(4):313.

13. Maragakis GM, Hahn P, Hellwig E. Chemomechanical caries removal: a comprehensive review of the literature. Int Dent J. 2001;51(4):291-9. pmid: 11570545.

14. Beeley JA, Yip HK, Stevenson AG. [Chemo-mechanical caries removal: a review of the techniques and latest developments]. Ned Tijdschr Tandheelkd. 2001;108(7):27781. pmid: 11486517.

15. Silva L, Motta L, Reda S, Facanha R, Bussadori S. Papacarie: A new system for the chemomechanical caries removal: case report. Rev Paul Odontol. 2004;26(6):4-8.

16. Pereira S, Silva L, Piccinini D, Santos E, Bussadori S Comparison antimicrobial potential in vitro between two materials for the Chemomechanical caries removal. 21th Annual Reunion SBPqO; Águas de Lindóia2004.

17. Ganesh M, Parikh D. Chemomechanical caries removal (CMCR) agents: Review and clinical application in primary teeth. J Dent Oral Hyg. 2011;3(3):34-45.

18. Habib C, Kronman J, Goldman M. A chemical evaluation of collagen and hydroxyproline after treatment with GK-101 (N-chloroglycine). Pharmacol Therapeut Dent. 1975;2(34):209-15.

19. Kleter GA, Damen JJ, Buijs MJ, Ten Cate JM. Modification of amino acid residues in carious dentin matrix. J Dent Res. 1998;77(3):488-95. doi: 10.1177/002203459807700308 01. pmid: 9496922.

20. Kotb RM, Abdella AA, El Kateb MA, Ahmed AM. Clinical evaluation of Papacarie in primary teeth. J Clin Pediatr Dent. 2009;34(2):117-23. pmid: 20297701. 